\title{
A characterization of the approximation order of multivariate spline spaces
}

by

AMOS RON* (Madison, Wis.)

Abstract. We analyze the approximation order associated with a directed set of spaces, $\left\{S_{h}\right\}_{h>0}$, each of which is spanned by the $h Z^{s}$-translates of one compactly supported function $\varphi_{h}: \mathbb{R}^{s} \rightarrow$ C. Under a regularity condition on the sequence $\left\{\varphi_{h}\right\}_{h}$, we show that the optimal approximation order (in the $\infty$-norm) is always realized by quasi-interpolants, hence in a linear way. These quasi-interpolants provide the best approximation rates from $\left\{S_{h}\right\}_{h}$ to an exponential space of good approximation order at the origin.

As for the case when each $S_{h}$ is obtained by scaling $S_{1}$, under the assumption

$$
\sum_{x \in Z^{*}} \varphi_{1}(\cdot-\alpha) \neq 0
$$

the results here provide an unconditional characterization of the best approximation order in terms of the polynomials in $S_{1}$. The necessity of (*) in this characterization is demonstrated by of the polynomials

1. Introduction. The determination of the approximation order associated with spline spaces is one of the major and fundamental goals of spline theory. In an abstract form, we are given a collection of function spaces $\left\{S_{h}\right\}_{h}$ where $h$ is a continuous or discrete positive parameter, and look for the largest $d$ for which

$$
\operatorname{dist}\left(f, S_{h}\right)=O\left(h^{d}\right), \quad \forall f \in F,
$$

where dist is measured by some metric or norm (usually a $p$ - or Sobolev norm) and $F$ is an admissible function space with respect to dist. Here, we always assume that all functions are complex-valued and defined on the vector space $\mathbf{R}^{s}$. We think of $h$ as measuring (in a linear way) the roughness of the mesh used, hence indeed expect to approximate better as $h \rightarrow 0$. A particular case of interest, which will be referred to as "the scaling case", occurs when the refined spaces $S_{h}$ are dilations of $S_{1}$, i.e.,

1985 Mathematics Subject Classification: Primary 41A15, 41A25, 41A40, 41A63; Secondary $65 \mathrm{D} 15$.

Key words and phrases: approximation order, Strang-Fix Conditions, exponentials, polynomials, multivariate, multivariate splines, uniform mesh, regular grids, integer translates, quasiinterpolation.

*Supported in part by Carl de Boor's Steenbock Professorship, University of Wisconsin-Madison and by the United States Army under Contract No, DAAL03-87-K-0030. 


$$
S_{h}=\sigma_{h} S_{1},
$$

with $\sigma_{h}$ the scaling operator

$$
\sigma_{h}: f \mapsto f(\cdot / h)
$$

In multivariate splines, as well as in finite elements, one prefers approximant spaces which are spanned by compactly supported functions, since then there is hope to provide a local scheme for approximation from the underlying space. In the area of multivariate splines on regular meshes, one also assumes that the spaces $S_{h}$ are translation-invariant, or more precisely, each $S_{h}$ is invariant under $h Z^{s}$-translates. This leads to the model where each of the spaces $S_{h}$ is assumed to be spanned by the $h \mathbf{Z}^{s}$-translates of one or finitely many compactly supported functions. In general, it is desirable to avoid the assumption that these translates are linearly independent, i.e., form a basis for $S_{h}$, since such condition is not met in many cases of practical interest (e.g., the Zwart element and also most of the cases when more than one compactly supported function is involved).

The simplest case therefore occurs when we assume both that $S_{1}$ is spanned by the integer translates of a single compactly supported function $\varphi$ and that $S_{h}$ is the $h$-scale of $S_{1}$, hence is spanned by the $h$-scale of $\varphi$. This model was investigated intensively in the late 60 's-early 70 's by people in the finite element area. The following theorem is essentially Theorem 1 of [SF] (at least for $p=2, \infty$ ) and these days is usually referred to as The Strang-Fix Conditions. In the statement of the theorem we use the notation $\varphi *_{h}$ for the semidiscrete convolution operator

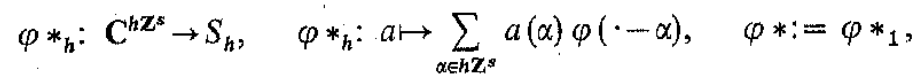

and $\varphi *_{h}^{\prime}$ for the semidiscrete operator

$$
\varphi *_{h}^{\prime} f:=\varphi *_{h}\left(\left.f\right|_{h \mathbf{Z}^{s}}\right), \quad \varphi *^{\prime}:=\varphi *_{1}^{\prime} .
$$

Also, $\Pi_{k}$ stands for the space of polynomials of (total) degree at most $k$.

(1.1) THEOREM. Let $\varphi$ be a compactly supported continuous function. Let $S_{1}$ be the space spanned by the integer translates of $\varphi$ and $S_{h}:=\sigma_{h} S_{1}$. Then for $1 \leqslant p \leqslant \infty$ the following conditions are equivalent:

(1) $\hat{\varphi}(0) \neq 0$, and for $|\alpha| \leqslant d-1, D^{\alpha} \hat{\varphi}$ vanishes on $2 \pi Z^{s} \backslash 0$.

(2) For every $\mu \in I_{d-1}, \varphi *^{\prime} \mu$ is a polynomial of the form $c \mu+q$, for some $c \neq 0$ and $q \in \Pi_{<\operatorname{deg} \mu}$.

(3) For every smooth function $f$ which is in $L_{p}$ together with all its derivatives up to order $d$, there exist sequences $a_{h}: h \mathbf{Z}^{s} \rightarrow \mathbf{C}$ satisfying

(3a) $\left\|f-\sigma_{h} \varphi *_{h} a_{h}\right\|_{p}=O\left(h^{d}\right)$.

(3b) $\left\|a_{h}\right\|_{l_{p}} \leqslant k h^{-s / p}\|f\|_{p}$.
The equivalence of (1) and (2) was refined and extended since then by several authors (cf. e.g., $\left[D M_{1}\right]$ and $\left[B_{1}\right]$; furthermore, $\left[B_{1}\right]$ shows that the first two conditions are equivalent to the apparently weaker one: $\hat{\varphi}(0) \neq 0$, $\Pi_{d-1} \subset S_{1}$ ), yet is mainly technical since it deals with a characterization of the polynomials in $S_{1}$ in terms of the Fourier transform $\hat{\varphi}$ and is not related directly to the approximation order. The implication $(1,2) \Rightarrow(3)$ initiated much work by many authors aiming at constructing explicit linear maps into $S_{h}$, the so-called quasi-interpolants, which satisfy the conditions in (3), and which (by now) are quite well understood (cf. $\left[\mathrm{B}_{2}\right]$ for an updated survey of quasi-interpolants). Still, it was probably the saturation-like result of the above theorem that was so spectacular: one cannot achieve approximation order $d$ from the dilated spaces $\left\{S_{h}\right\}_{h}$ unless $\Pi_{d-1} \subset S_{1}$ ! However, one should bear in mind that condition (3) is assuming a restrictive way for approximation from $S_{h}$ (termed "controlled"), hence does not characterize the approximation order from $\left\{S_{h}\right\}_{h}$ for. smooth functions, and gives only a lower bound for that approximation order. As a matter of fact the desired approximation order from spaces of the above form is known only in some special cases (such as when $\varphi$ is a box spline, $\left[\mathrm{BH}_{1}\right]$ ). Replacing the "controlled" notion by another constrained approximation notion introduced in [BJ] (and referred there to as "local approximation"), Theorem (1.1) admits a generalization to the case of several compactly supported functions ([SF], [BJ]), however still leaving open the question of the unconditional approximation order. Finally, the analysis in [DR] (of the approximation order for exponential box spline spaces) demonstrated the fact that good rates of approximation can be obtained from spaces $\left\{S_{h}\right\}$, where each one of them is spanned as before by the translates of a single function, yet none of them contains nontrivial polynomials. (Clearly, such spaces are not obtained by dilating $S_{1}$ ). In view of these latter results, one may interpret the Strang-Fix Conditions as saying that constructing $S_{h}$ by scaling $S_{1}$ is appropriate only when $S_{1}$ contains a sufficiently large space of polynomials.

In this paper, we characterize the order of best approximation from spaces of the form $\left\{S_{h}:=a_{h} S_{1}\right\}_{h}$, with $S_{1}$ spanned by the integer translates of a compactiy supported function $\varphi$. We show that, under the assumption

$$
\sum_{\alpha \in B^{*} Z^{\infty}} \varphi(\cdot-\cdots) \neq 0 \text {, }
$$

the approximation order associated with $\left\{S_{h}\right\}_{h}$ is determined by the polynomials in $S_{1}$ exactly in the same way the controlled and local approximation orders are characterized, thus improving the statement of the Strang-Fix Conditions above. Our course, though, does not focus on (and as a matter of fact is not aimed at) spaces obtained by scalings, but considers the general setting of an arbitrary directed function space set $\left\{S_{h}\right\}_{h}$, each of which is spanned by $h \mathbf{Z}^{s}$-translates of a compactly supported function $\varphi_{h}$. The goal is to provide information about the approximation order of $\left\{S_{h}\right\}_{h}$ and about maps 
that realize this approximation order. In order to make a good use of the compact support assumption, it is essential to assume that for some $k>0$

$$
\operatorname{supp} \varphi_{h} \subset[0, h k]^{s}, \quad \forall h,
$$

since otherwise the translates of $\varphi_{h}$ are not uniformly local as $h \rightarrow 0$.

A major step in the characterization here of the above-mentioned approximation order is the construction, for a given finite-dimensional space $H$ of a certain type, of a sequence $\left\{Q_{h}\right\}_{h}$ of uniformly local, uniformly bounded quasi-interpolants (each maps into the associated $S_{h}$ ), that approximates the elements of $H$ to the same order as best approximations do. In that process $H$ is required to be a translation-invariant subspace of $L_{(x)}$, which leads naturally to exponential spaces $H$ spanned by exponentials of imaginary frequencies. Using quasi-interpolation arguments, we show that the operators $\left\{Q_{h}\right\}_{h}$ provide approximants of optimal order to all admissible functions, in case $H$ approximates well locally (say, at the origin). This gives rise to a scheme for the determination of the approximation order from $\left\{S_{h}\right\}_{h}$, in which finding this order is reduced to determining the approximation order obtained by applying a prescribed set of quasi-interpolants to prescribed (and finitely many) exponentials. This also proves that the best approximation order can always be realized in a linear way (i.e., by a quasi-interpolant), and furthermore, that we can construct quasi-interpolants of optimal order (essentially) without an a priori (or even a posteriori) knowledge about this approximation order.

In the special case when $\varphi_{h}=\sigma_{h} \varphi_{1}$, the quasi-interpolants constructed are not scales one of the other (i.e., $Q_{h} \neq \sigma_{h} Q_{1} \sigma_{1 / h}$ ), yet it is shown that the scaled operators $\left\{\sigma_{1 / h} Q_{h}\right\}_{h}$ converge, and the limiting quasi-interpolant $Q$ is then used to obtain the above-mentioned improvement of the Strang-Fix Conditions.

In Section 2 we analyze first the relatively simpler case of approximation order to smooth bounded functions, an analysis which serves as an illustration of the general case, while being better tight in its statements and error estimates. The main results together with their proofs are given in Section 3. Finally, in Section 4 we consider the scaling case under the singularity assumption $\sum_{\alpha E Z^{s}} \varphi(\alpha)=0$. We prove a theorem which allows us to embed (in a suitable sense) the case of several $\varphi$ 's (as studied in $[\mathrm{SF}],\left[\mathrm{DM}_{2}\right],[\mathrm{J}]$ and $[\mathrm{BJ}])$ in this case of a single singular $\varphi$, and thus to use the counterexample of $\left[\mathrm{BH}_{2}\right]$ to show that the polynomials in $S_{1}$ do not characterize any more the approximation order, leaving perhaps only little hope for a clear and simple characterization of the approximation order for this more general setting.

We use standard multivariate notations. So $\mathbf{Z}_{+}^{s}:=\left\{\alpha \in \mathbf{Z}^{s}: \alpha \geqslant 0\right\}$, and for $\alpha \in \mathbf{Z}_{+}^{s}, 0^{\alpha}$ is the power function $x \mapsto x^{\alpha}:=x_{1}^{\alpha_{1}} \ldots x_{s}^{\alpha_{s}}, D$ and $E$ are used for the differential resp. shift operators, and so $p(D)$ and $p(E)$ are the differential resp. difference operators obtained by evaluating the polynomial $p$ at $D$ and $E$; in particular, $E^{\alpha}: f \mapsto f(\cdot+\alpha)$.
One concluding remark seems to be in order: the Strang-Fix Conditions, as well as their proofs, strongly emphasize the Fourier analysis approach (Poisson's summation, Parseval formula). Through the years it led to the understanding that the Fourier analysis methods are essential to the characterization of approximation orders and related issues, at least for general uniform spaces. The results here, as well as their proofs, provide an illustration of the alternative techniques: while the Fourier transform approach focuses (implicitly) on the similarity between the semidiscrete operator $\varphi *^{\prime}$ and the standard convolution operator $\varphi *$ for suitable spaces of polynomials or exponentials (see $\left[\mathrm{BR}_{3}\right]$ which elaborates on this point), the alternative approach (as originated in $\left[B_{1}\right]$, with the results in [CJW] and [CD] serving as the motivation) associates $\varphi *^{\prime}$ with the difference operator

$$
T_{\varphi p}: f \mapsto \sum_{\alpha \in Z^{s}} \varphi(\alpha) f(\cdot-\alpha)
$$

In this setting, invariant subspaces of $S_{h}$ (under differentiation, $h \mathbf{Z}^{s}$-translations, etc.) play a key role. The above difference operators match the action of $\varphi *^{\prime}$ for more general spaces other than polynomials or exponentials $\left(\left[\mathrm{B}_{1}\right],\left[\mathrm{R}_{1}\right]\right)$, hence are adequate for the analysis here. Moreover, even for piecewisepolynomials or piecewise-exponentials this approach is sometimes more direct and -more efficient than Fourier analysis (cf. e.g., $[\mathrm{BAR}]$ and $\left[\mathrm{R}_{2}\right]$ ).

2. Approximation order for continuous functions. In this section we illustrate the general results by treating first the approximation order for bounded uniformly continuous functions.

Throughout this section, as well as in the next one, we assume that $\left\{\varphi_{h}\right\}_{h}$ is a collection of compactly supported functions which satisfy, for every $h$, the following three conditions:

$$
\begin{gathered}
\operatorname{supp} \varphi_{h}=[0, h k)^{s} \\
\sum_{\alpha \in h Z^{*}} \varphi_{h}(\alpha)=1 \\
\left\|\varphi_{h}\right\|_{h x} \leqslant c
\end{gathered}
$$

Here $k$ and $c$ are some constants, which will be used in the sequel in the above meaning without further reference. Note that $\not h\left(h \mathbf{Z}^{s} \cap \operatorname{supp} \varphi_{k}\right) \leqslant k^{s}$. Wo also use the notation

$$
\operatorname{dist}(f, S):=\inf \left\{\|f-g\|_{\infty x}: g \in S\right\} .
$$

(2.5) Propositron. For every $h$,

$$
\left\|\varphi_{h} *_{h}^{\prime} 1-1\right\|_{\infty} \leqslant 2 k^{s} \text { c dist }\left(1, S_{h}\right) \text {. }
$$

Proof. Let $f$ be an arbitrary element of $S_{n}$. Then, for every $\alpha \in \mathbf{R}^{s}$, 
Since $f \in S_{h}$, we know from $\left[\mathrm{B}_{1}\right]$ that $\varphi_{h} *_{h}^{\prime} f=\sum_{\alpha=h \mathbf{Z}^{s}} \varphi_{h}(\alpha) E^{-\alpha} f$, and hence, by (2.1), (2.2) and (2.7),

$$
\begin{aligned}
\left\|\varphi_{h} *_{h}^{\prime} f-1\right\|_{\infty} & =\left\|\sum_{\alpha \in h \mathbb{Z}^{s}} \varphi_{h}(\alpha)\left(E^{-\alpha} f-1\right)\right\|_{\infty} \\
& \leqslant \sum_{\alpha \in h Z^{s}}\left|\varphi_{h}(\alpha)\right|\left\|E^{-\alpha} f-1\right\|_{\infty} \leqslant k^{s} c\|f-1\|_{\alpha} .
\end{aligned}
$$

But this completes the proof, since (2.1), when combined with (2.3), implies that

$$
\left\|\varphi_{h} *_{h}^{\prime}(f-1)\right\|_{\infty} \leqslant k^{s} c\|f-1\|_{\infty} .
$$

The above result is now used in the following theorem, which shows that the operators $\left\{\varphi_{h} *_{h}^{\prime}\right\}_{h}$ approximate continuous functions to the same order as the best approximations from $\left\{S_{h}\right\}_{h}$ do.

(2.8) THEOREM. Assume that (2.1)-(2.3) hold. Then for every function $f$ and every $h$,

$$
\left\|\varphi_{h} *_{h}^{\prime} f-f\right\|_{\infty} \leqslant c k^{s}\left(k \omega_{f}(h)+2 \operatorname{dist}\left(1, S_{h}\right)\|f\|_{\infty}\right),
$$

where $\omega_{f}$ is the modulus of continuity of $f$ (in the $\infty$-norm). In particular, if $\operatorname{dist}\left(1, S_{h}\right)=o(1)$, then $\operatorname{dist}\left(f, S_{h}\right)=o$ (1) for every bounded uniformly continuous $f$, and if dist $\left(1, S_{h}\right)=O(h)$, then dist $\left(f, S_{h}\right)=O(h)$ for every $f \in C^{1}\left(\mathbf{R}^{s}\right)$ which is bounded together with its first order derivatives.

Proof. Fix $h$ and $x \in \mathbf{R}^{s}$. Since, by $(2.1), \varphi_{h}(x-\alpha)=0$ for all $\alpha \notin x-[0, h k)^{s}$, Proposition (2.5) implies that, with $v_{h}(x):=h \mathbf{Z}^{s} \cap\left(x-\operatorname{supp} \varphi_{h}\right)$,

$$
\left|\sum_{\alpha \in v_{h}(x)} \varphi_{h}(x-\alpha)-1\right| \leqslant 2 k^{s} c \operatorname{dist}\left(1, S_{h}\right)
$$

and hence

$$
\left|\sum_{\alpha \in \psi_{h}(x)} f(x) \varphi_{h}(x-\alpha)-f(x)\right| \leqslant 2 k^{s} c \operatorname{dist}\left(1, S_{h}\right)\|f\|_{\infty} .
$$

On the other hand, by $(2.3)$,

$$
\text { (2.12) } \begin{aligned}
\mid \sum_{\alpha \in v_{h}(x)} f(\alpha) \varphi_{h}(x-\alpha) & -\sum_{\alpha \in v_{h}(x)} f(x) \varphi_{h}(x-\alpha) \mid \\
& \leqslant \sum_{\alpha \in v_{h}(x)}|f(\alpha)-f(x)|\left|\varphi_{h}(x-\alpha)\right| \leqslant c k^{s+1} \omega_{f}(h) .
\end{aligned}
$$

Summing (2.11) and (2.12) we obtain (2.9), which implies the rest of the theorem.

Theorem (2.8) leads to the following improvement of the case $d=0$ (and $p=\infty)$ in Theorem (1.1):

(2.13) COROLLARY. Let $\varphi$ be a bounded compactly supported function satisfying $\sum_{\alpha \in \mathbf{Z}^{s}} \varphi(\alpha)=1$. Define $\varphi_{h}:=\sigma_{h} \varphi$, and let $S_{h}$ be the space spanned by the $h \mathbf{Z}^{s}$-translates of $\varphi_{h}$. Then the following conditions are equivalent: (a) dist $\left(f, S_{h}\right)=O(h)$, for every $f \in C^{1}\left(\mathbf{R}^{s}\right)$ which is bounded together with its first order derivatives.

(b) For every function $f$

$$
\left\|\varphi_{h} *_{h}^{\prime} f-f\right\|_{\infty} \leqslant M \omega_{f}(h)
$$

where $M$ depends only on $\operatorname{diam} \operatorname{supp} \varphi$ and $\|\varphi\|_{\infty}$.

(c) $1 \in S_{1}$.

(d) $\varphi *^{\prime} 1=1$.

Proof. (d) $\Rightarrow$ (c). Trivial.

(c) $\Rightarrow$ (b). (2.2) is assumed here and (2.1) and (2.3) are trivially satisfied, and therefore Theorem (2.8) can be invoked. (b) then follows from the fact that, since $1 \in S_{1}, 1 \in S_{h}$ for every $h$, and thus dist $\left(1, S_{h}\right)=0$.

(b) $\Rightarrow$ (a). Trivial.

(a) $\Rightarrow$ (d). With $f=1$, we employ Proposition (2.5) to conclude that $\| \varphi_{h} *_{h}^{\prime} 1$ $-1 \|_{\infty} \rightarrow 0$ as $h \rightarrow 0$. But since $\left\|\varphi_{h} *_{h}^{\prime} 1-1\right\|_{\infty}$ is independent of $h$, it must be 0 .

3. Approximation order for smooth functions. In this section we establish necessary and sufficient conditions for higher approximation orders (i.e., higher than $O(h)$ ) for smooth functions by the spaces $\left\{S_{h}\right\}_{h}$. Precisely, we look for the maximal $d$ for which dist $\left(f, S_{h}\right)=O\left(h^{d}\right)$ for every function in the Sobolev space

$$
W_{\infty}^{d}:=\left\{f \in C^{d}\left(\mathbf{R}^{y}\right):\|f\|_{\infty, d}:=\sum_{|\alpha| \leqslant d}\left\|D^{\alpha} f\right\|_{\infty}<\infty\right\} .
$$

Throughout the section we retain assumptions (2.1)-(2.3).

The proofs in the previous section were based on the fact that the sequence $\left\{\varphi_{h} *^{\prime}\right\}$, forms a collection of uniformly bounded local operators which approximate continuous functions to the same order as the best approximation. The constant function is a convenient trial function since on the one hand it spans a translation-invariant space while on the other hand it is admissible, being bounded and uniformly continuous. In the general case, though, the operator $\varphi_{h} *_{h}^{\prime}$ cannot be expected to approximate well, and will be replaced by an operator of the form $\psi_{h} \psi_{h}^{\prime}$ for a suitable function $\psi_{h} \in S_{k}$. Furthermore, we avoid here polynomials as trial functions, since in general they are neither bounded nor uniformly continuous. Seeking for finite-dimensional spaces $H$ which are translation-invariant on the one hand, and consist of bounded functions on the other hand, we note that such spaces are necessarily of the form

$$
H=\operatorname{span}\left\{e_{\theta}\right\}_{\theta \in \Theta},
$$

where $e_{\theta}: x \mapsto e^{\theta \cdot x}$, and the spectrum $\Theta$ of $H$ satisfies

$$
\Theta \subset i \mathbf{R}^{s} \text {. }
$$

The first result here deals with the extension of Proposition (2.5) to exponential spaces $H$ of the above form, and is the crux in the analysis to follow. We use here the notation $T_{h}$ for the difference operator 


$$
T_{h}: f \mapsto \sum_{\alpha \in h \mathbf{Z}^{s}} \varphi_{h}(\alpha) E^{-\alpha} f .
$$

(3.5) THEOREM. Assume $\left\{\varphi_{h}\right\}_{h}$ satisfy (2.1)-(2.3). Let $H$ be a finite-dimensional exponential space satisfying (3.2) and (3.3). Then there exists a sequence $\left\{V_{h}\right\}_{h}$ of difference operators, supported on $h \mathbf{Z}^{s}$ (resp.) and satisfying

(a) $V_{h}$ inverts $\left.T_{h}\right|_{H}$ for all (sufficiently small) $h$;

(b) $\left\{V_{h}\right\}_{h}$ are uniformly local and uniformly bounded, i.e.,

$$
\operatorname{supp} V_{h} \varphi_{h} \subset\left[0, h k_{1}\right)^{s}, \quad\left\|V_{h} \varphi_{h}\right\|_{\alpha,} \leqslant c_{1},
$$

for some constants $k_{1}, c_{1}$.

The functions $\psi_{h}:=V_{h} \varphi_{h}$ then satisfy

$$
\left\|\psi_{h} *_{h}^{\prime} f-f\right\|_{\infty} \leqslant M \operatorname{dist}\left(f, S_{h}\right)
$$

for every $f \in H$. The constant $M$ depends only on $k, c$ and $\varrho(H):=\max \left\{\|0\|_{\infty}\right.$ : $\left.e_{0} \in H\right\}$.

(3.8) Remark. The representation, hence the extension, of $\left(\left.T_{h}\right|_{H}\right)^{-1}$ as difference operators in the approach here is merely a convenient choice. Any uniformly bounded uniformly local extensions will do as well.

Proof. Set $\Theta$ for the spectrum of $H$. With $T_{h}$ as in (3.4), (2.3) and (2.1) imply that

$$
\left\|T_{h}\right\| \leqslant c k^{s},
$$

when regarding ${ }^{\prime} T_{h}$, say, as an endomorphism of $L_{\infty}\left(\mathbf{R}^{s}\right)$. We claim that for sufficiently small $h$, the restrictions $\left.T_{h}\right|_{H}$ are equibounded below by some positive constant. We use it to define the difference operators $\left\{V_{h}\right\}_{h}$, which are uniformly bounded (at least for small enough $h$ ) and invert $\left\{T_{h}\right\}_{\text {n }}$ on $H$ (as done in $[D R]$ in the derivation of the approximation order for exponential box splines. The idea, for a polynomial $H$ and $h=1$, was originated in [CD]). First, we examine the action of $T_{h}$ on the pure exponentials of $H$ : for an exponential $e_{\theta}, T_{h}\left(e_{\theta}\right)=e_{\theta} \sum_{y_{h}(0)} \varphi_{h}(\alpha) e^{-\theta \cdot \alpha}$ (with $v_{h}(0):=h \mathbf{Z}^{s} \cap[0, h k)^{s}$ ), while

(3.10) $\left|\sum_{v_{h}(0)} \varphi_{h}(\alpha) e^{-\theta \cdot \alpha}\right| \geqslant\left|\sum_{\nu_{h}(0)} \varphi_{h}(\alpha)\right|-\left|\sum_{\nu_{h}(0)} \varphi_{h}(\alpha)\left(1-e^{-\theta \cdot \alpha}\right)\right| \geqslant 1-k^{s} c b h$,

using (2.1)-(2.3). Here $b$ depends on $\varrho(H)$ and $k$ but is independent of $h$, which means that (for sufficiently small $h$ and) for all $e_{\theta} \in H$

$$
T_{h} e_{\theta}=c_{h, \theta} e_{\theta} \text {, where }\left|c_{h, \theta}\right| \geqslant 1 / 2 \text {. }
$$

We now define

$$
V_{h}:=\left(I-\prod_{\theta \in \Theta}\left(I-\left(1 / c_{h, \theta}\right) T_{h}\right)\right) / T_{h},
$$

with $I$ being the identity (the division by $T_{h}$ here is formal, since $T_{h}$ appears as a factor in the numerator of the right hand of (3.12)). Since, for each $\theta$,
$I-\left(1 / c_{h, \theta}\right) T_{h}$ annihilates $e_{\theta}$, the product term in the definition of $V_{h}$ annihilates $H$, and hence, for every $h,\left.\left(V_{h} T_{h}\right)\right|_{H}=I$, which proves (a)

As for the functions $\left\{\psi_{h}\right\}_{h}$ in the theorem, with the aid of (3.9) and (3.11), we see that $\left\{V_{h}\right\}_{h}$ is a bounded set of operators, and since each $V_{h}$ is a linear combination of $\left.\left\{T_{h}^{j}\right\}\right\}_{j=0}^{* \theta-1}$, it is a finite difference operator supported on $[0, h r)^{s} \cap h \mathbf{Z}^{s}$ (where $r \leqslant k \# \Theta$ ), and hence (b) is valid.

To prove (3.7), we now take any $f \in H$ and an arbitrary $f_{h} \in S_{h}$. Since

$$
\left\|\psi_{h} *_{h}^{\prime} f-f^{\prime}\right\|_{\infty} \leqslant\left\|\psi_{h} *_{h}^{\prime} f-V_{h} T_{h} f_{h}\right\|_{\infty}+\left\|V_{h} T_{h} f_{h}-f\right\|_{\infty},
$$

it is sufficient to estimate each of the right-hand summands in (3.13).

As for the first one, we recall that since $f_{h} \in S_{h}$ we have, by $\left[\mathrm{B}_{1}\right]$, $T_{h} f_{h}=\varphi_{h} *_{h}^{\prime} f_{h}$ and hence $V_{h} T_{h} f_{h}=\psi_{h} *_{h}^{\prime} f_{h}$. Therefore

(3.14) $\quad\left\|\psi_{h} *_{h}^{\prime} f-V_{h} T_{h} f_{h}\right\|_{\infty}=\left\|\psi_{h} *_{h}^{\prime}\left(f-f_{h}\right)\right\|_{\infty} \leqslant k_{1}^{s} c_{1}\left\|f-f_{h}\right\|_{h}$

For the second term, we use the fact that $\dot{V}_{h}$ inverts $T_{h}$ on $H$, hence

$$
\left\|V_{h} T_{h} f_{h}-f\right\|_{\infty}=\left\|V_{h} T_{h}\left(f_{h}-f\right)\right\|_{\infty} \leqslant\left\|V_{h} T_{h}\right\|\left\|f_{h}-f\right\|_{\infty} .
$$

Summing (3.14) and (3.15), and in reliance on the boundedness of $\left\{V_{h} T_{h}\right\}_{h}$, we obtain

$$
\left\|\psi_{k} *_{h}^{\prime} f-f\right\|_{\infty} \leqslant M\left\|f-f_{h}\right\|_{\infty},
$$

which implies (3.7), since $f_{h}$ was arbitrary.

For later use, we examine in the following corollary the above construction under the scaling assumption.

(3.16) COROLlary. Under the assumption $\varphi_{h}=\sigma_{h} \varphi_{1}$, all $h$, we have

$$
\sigma_{1 / h} \psi_{h} \rightarrow\left(\left(I-\left(I-T_{1}\right)^{* \theta}\right) / T_{1}\right) \varphi_{1} \quad \text { as } h \rightarrow 0,
$$

where $\left\{\psi_{h}\right\}_{h}$ are the functions constructed in Theorem (3.5). The convergence is uniform.

Proof. Using the notations of Theorem (3.5) we have

$$
\sigma_{1 / h} \psi_{h}=\sigma_{1 / h} V_{h} \sigma_{h} \varphi_{1} .
$$

Furthermore, the assumption $\varphi_{h}=\sigma_{h} \varphi_{1}$ also implies that $\sigma_{1 / h} T_{h} \sigma_{h}=T_{1}$, and the claim then follows from the fact that the constants $c_{h, \theta}$ involved in the definition of $V_{h}$ satisfy $\lim _{\left.h h_{*}\right)} c_{h, \theta}=1, \forall \theta \in \Theta((3.10)$ bounds this limit from above by 1 , and a similar argument provides the converse inequality).

The proofs of the main results make use of the "local approximation order" (1) notion (as do all quasi-interpolation arguments). As a preparation,

( $\left.{ }^{1}\right)$ Unfortunately, the terminology "local approximation" is used in spline theory in two different contexts: the "local approximation" mentioned in the introduction in conjuction with $[\mathrm{BJ}]$ is thus a different notion. 
we define now the local approximation order (or the "approximation order at a point"), and discuss some aspects of the local order which are relevant to the context here.

(3.17) Definition. Let $H$ be a subspace of $C^{\infty}\left(\mathbf{R}^{s}\right)$. We say that $H$ admits a local approximation order $d$ at $x \in \mathbf{R}^{s}$ if for every $f$ which is sufficiently smooth in a neighborhood of $x$, there exists $g \in H$ such that

$$
|(f-g)(x+y)| \leqslant c_{f, x}\|y\|_{i x}^{d},
$$

for sufficiently small $y$

Note that in case $H$ is translation-invariant, the local approximation order of $H$ is independent of $x$. For an exponential $H$ of the form (3.2), the results of $\left[\mathrm{BR}_{1}\right]$ provide a useful characterization of the local approximation order.

(3.19) Result. Assume $H$ satisfies (3.2). Then the local approximation order $d$ of $H$ is determined by the largest polynomial space $\Pi_{d-1}$, for which the spectrum of $H$ is total.

We recall that a set $\Omega$ is total for the function space $F$ if no $f \in F \backslash 0$ vanishes identically on $\Omega$.

For our purposes, it is also important to have some bounds on the approximant $g$ in terms of the Sobolev norm $\|f\|_{\alpha, d}$. This task is accomplished in the following proposition.

(3.20) Proposition. Let $H$ be a finite-dimensional exponential space of local approximation order $d$, which satisfies (3.2) and (3.3). Then, for every $f \in W_{\infty}^{d}$ and every $x \in \boldsymbol{R}^{s}$, the local approximant $g:=\sum_{\theta \in \Theta} c_{\theta} e_{\theta} \in H$ can be chosen such that

$$
\begin{aligned}
&|(f-g)(x+y)| \leqslant \text { const. }\|f\|_{\alpha, d}\|y\|_{i_{\infty}}^{d}, \\
& \max _{\theta \in \Theta}\left|c_{\theta}\right| \leqslant \text { const. }\|f\|_{\infty, d, d}
\end{aligned}
$$

where const. depends only on $H$.

Proof. The inequality (3.21) follows as a special case of the argument used in the proof of Theorem 3.1 in [DR]. As for the second claim, in view of Result (3.19), we may assume without loss that $\# \Theta=\operatorname{dim} I_{d \ldots 1}$, since otherwise $H$ can be replaced by a subspace of it of dimension $\operatorname{dim} \Pi_{d \rightarrow 1}$ and with spectrum $\Theta^{\prime}$ which is still total for $\Pi_{d-1}$.

Now fix $x \in \boldsymbol{R}^{s}$ and $f \in W_{x,}^{d}$. The assumption on the local approximation order property of $H$ can now be combined with the fact that $\operatorname{dim} H=\operatorname{dim} \Pi_{d-1}$ to conclude that the local approximant $g \in H$ for $f$ at $x$ is unique. This means that the seminorm $\|g\|^{\prime}:=\max _{|\alpha|<d}\left|D^{\alpha} g(x)\right|$ is a norm on $H$, hence is equivalent to the norm $\left\|\sum_{0 \in \Theta} c_{\theta} e_{\theta}\right\|^{\prime \prime}:=\max _{\theta \in \Theta}\left|c_{\theta}\right|$. Since $\|g\|^{\prime}=\|f\|^{\prime}$. we obtain (3.22). The fact that the constant there is uniform in $x$ follows easily from the fact that $\left|e_{\theta}(x)\right|=1$ for every $\theta \in \Theta$ and $x \in \mathbf{R}^{s}$.
(3.23) Tylorim. Suppose that $\left\{\varphi_{h}\right\}_{\text {satisfy }}(2.1)-(2.3)$. Let $H$ be a finitedimensional exponential space that satisfies (3.2) and (3.3) and is of local approximation order $d$. Let $\left\{\psi_{h}\right\}_{h}$ be the functions associated with $H$ by Theorem (3.5). Then, for some constants $M_{1}, M_{2}$ and for every $f \in W_{\infty}^{d}$,

$$
\begin{aligned}
& \left\|\psi_{h} * *_{h}^{\prime} f-f\right\|_{1, z} \leqslant\|f\|_{1 x, d}\left(M_{1} h^{d}+M_{2} \max \operatorname{dist}\left(e_{\theta}, S_{h}\right)\right) \text {. } \\
& \text { entell }
\end{aligned}
$$

Proof. Let $f \in W_{x}^{d}$ and $x \in \mathbf{R}^{x}$. Choose $g:=\sum c_{\theta} e_{0} \in H$ to be as in Proposition (3.20). Then, by Proposition (3.20) and Theorem (3.5),

Therefore,

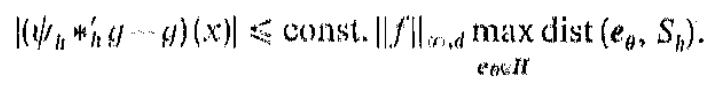

$$
\begin{aligned}
& \left|\left(\psi_{h} *_{h}^{\prime} f-f\right)(x)\right| \leqslant\left|\psi_{h} *_{h}^{\prime}(f-g)(x)\right|+\left|\left(\psi_{h} * *_{h}^{\prime} g-g\right)(x)\right|
\end{aligned}
$$

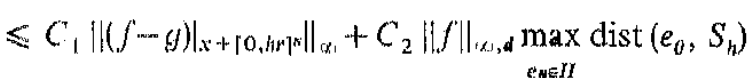

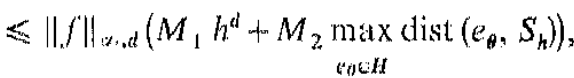

where in the last inequality the fact that $g(x)=f(x)$ has been used.

Combining Result (3.19) with Theorem (3.23) we conclude the following:

(3.25) Coroulary. Suppose that $\left\{\varphi_{h}\right\}_{h}$ salisfy (2.1)-(2.3). Then the following conditions are equivalunt:

(a) For every f $\in W_{i \neq}^{d}, \operatorname{dist}\left(f, S_{h}\right)=O\left(h^{d}\right)$.

(b) For any (every) finite $\Theta \subset i \mathbf{R}^{s}$ which is total for $\Pi_{d-1}$, the functions $\left\{\psi_{n}\right\}_{h}$ constructed in Theorem (3.5) with respect to $H:=\operatorname{span}\left\{e_{\theta}\right\}_{\theta \in \Theta}$ satisfy. $\left\|\psi_{h} *_{h}^{\prime} e_{0}-e_{0}\right\|_{i x}=O(h)^{\prime}$, for enery ()$\in \Theta$.

The above corollary gives rise to the following scheme for the determination of the approximation order from the directed family of spaces $\left\{S_{h}\right\}_{h}$ :

(3.26) SchHiml. (hoises

$$
\Theta 1, \omega, \ldots \in i \mathbf{R}^{s}
$$

such that each $\omega_{1}$ is tolal for $H_{d \cdots 1}$ and define $H_{d}$ : $d=1,2, \ldots d l$ :

Step 1: Chock your provious workefe of approximation orders, if one of these is $d$, then stop. $d$ is your approximation order. (Skip this step for $d=1$ ).

Step 2: Construct the quasi-interpolants $\left\{\psi_{b} *_{h}^{\prime}\right\}_{4}$ (with respect to $H_{d}$ ).

Step 3: For each $0 \in \omega_{d} \backslash \omega_{d}$. determine the order of $\left\|\psi_{h} *_{h} e_{0}-e_{0}\right\|_{\infty}$. If one of these rates is d, stop. dis then you approximation order. Otherwise store the smallest rate oblained hare, increase of by 1 and go to step 1. At this point you already know that the approximation order is at least $d+1$, and does not exceed the least of the orders stored. 
One way to choose the sets $\left\{\Theta_{d}\right\}_{d}$ is

$$
\Theta_{d}=\left\{i \alpha: \alpha \in \mathbf{Z}_{+}^{s},|\alpha| \leqslant d-1\right\}
$$

It should be emphasized that in practice the above results do not necessarily require the identification of the approximation order for constructing good quasi-interpolants. Choosing $H$ of local approximation $d$, we may use $\left\{\psi_{h}\right\}_{h}$ of Theorem (3.5), knowing that the order of the scheme would be $\min \left\{d, d_{0}\right\}$ where $d_{0}$ is the (presumably unknown) approximation order.

The last result of this section provides the following improvement of Theorem (1.1) (for $p=\infty$ ):

(3.27) THEOREM. Let $\varphi$ be a compactly supported bounded function satisfying $\sum_{\alpha \in \mathbf{Z}^{s}} \varphi(\alpha) \neq 0$. Define $\varphi_{h}:=\sigma_{h} \varphi$, and $S_{h}:=\operatorname{span}\left\{E^{\alpha} \varphi_{h}\right\}_{\alpha \in h \mathbf{Z}^{s}}$. Then the following conditions are equivalent:

(a) For every function $f \in W_{\infty}^{d}$

$$
\operatorname{dist}\left(f, S_{h}\right)=O\left(h^{d}\right)
$$

(b) $\Pi_{d-1} \subset S_{1}$.

Proof. The implication (b) $\Rightarrow$ (a) is well known (and by now standard). For the converse, note first that we may assume without loss that $\varphi$ is normalized to satisfy (2.2). We then choose $\Theta$ to be a finite subset of $i \mathbf{R}^{s}$ which is total for $\Pi_{d-1}$ and define $H:=\operatorname{span}\left\{e_{\theta}\right\}_{\theta \in \theta \cdot}$. Then, since $H \subset W_{\infty}^{d}$, dist $\left(f, S_{h}\right)=O\left(h^{d}\right)$ for every $f \in H$ and hence Theorem (3.23) (when combined with Result (3.19)) implies that the $\left\{\psi_{h}\right\}_{h}$ constructed in Theorem (3.5) (with respect to the prem sent $H$ ) satisfy

$$
\left\|\psi_{h} *_{h}^{\prime} f-f\right\|_{\infty}=O\left(h^{d}\right)
$$

for every $f \in W_{\infty}^{d}$. Rescaling each $\psi_{h}$ back to the original mesh size we obtain a sequence of functions $\left\{g_{h}:=\sigma_{1 / h} \psi_{h}\right\}_{h} \subset S_{1}$, which, by Corollary (3.16), converges uniformly to some $g \in S_{1}$.

Now, choose $f \in W_{\infty}^{d}$ which coincides with a homogeneous polynomial $p$ of degree $k$ in a neighborhood of the origin. Then, for a fixed $x \in \mathbf{R}^{s}$ and sufficiently small $h$,

$$
\begin{aligned}
h^{k}\left|\left(g_{h} *^{\prime} p-p\right)(x)\right| & =h^{k}\left|\left(\psi_{h} *_{h}^{\prime} \sigma_{h} p\right)(h x)-p(x)\right|=\left|\left(\psi_{h} *_{h}^{\prime} p-p\right)(h x)\right| \\
& =\left|\left(\psi_{h} *_{h}^{\prime} f-f\right)(h x)\right| \leqslant\left\|\psi_{h} *_{h}^{\prime} f-f\right\|_{1}=O\left(h^{d}\right) .
\end{aligned}
$$

Thus, in case $k \leqslant d-1$, we obtain

$$
\left(g *^{\prime} p-p\right)(x)=\lim _{h \rightarrow 0}\left(g_{h} *^{\prime} p-p\right)(x)=0 .
$$

We conclude that $g *^{\prime} p=p$ for every homogeneous $p \in \Pi_{d-1}$, hence for every $p \in \Pi_{d-1}$, and since $g \in S_{1}, \Pi_{d-1} \subsetneq S_{1}$ as well.

4. Singularity and the case of several compactly supported functions. Our primary aim in this section is to obtain results concerning the approximation order in case $\varphi:=\varphi_{1}$ satisfies the singularity condition

$$
\sum_{\alpha \in \mathbf{Z}^{s}} \varphi(\alpha)=0,
$$

and the refined spaces $\left\{S_{h}\right\}$ are obtained by scaling. The questions we focus on are whether good approximation rates are possible at all in such a situation (answer: yes), and whether the approximation order is determined by the polynomials in $S(\varphi):=S_{1}(\varphi)$ in the same way as in the regular case (answer: in general, no).

Some of the methods employed here are aimed to connect the case of a single $\varphi$ to the case of many $\varphi$ 's. In doing so, we obtain several results on the latter case which are of independent interest.

Throughout this section, $\varphi$ is a compactly supported bounded function, $S(\varphi)$ is the space spanned by the integer translates of $\varphi$, and the spaces $\left\{S_{h}\right\}_{h}:=\left\{S_{h}(\varphi)\right\}_{h}$ are obtained by scaling $S(\varphi)$, i.e., $S_{h}=\sigma_{h} S(\varphi)$.

We first show that (4.1) dashes any hope for a positive approximation order, unless the stronger assumption

$$
\varphi *^{\prime} 1=0
$$

is made:

(4.3) Proposition. Assume that (4.1) holds, yet $\varphi *^{\prime} 1 \neq 0$. Then dist $\left(1, S_{h}\right)$ $\neq O(h)$.

Proof. Since 1 is translation-invariant, dist $\left(1, E^{x} S_{h}\right)=\operatorname{dist}\left(1, S_{h}\right)$, for every $h$ and every real translation $E^{x}$ of $S_{h^{*}}$ On the other hand, since $\varphi *^{\prime} 1 \neq 0$, one can find a translate $\psi:=E^{x} \varphi$ which satisfies the regularity condition $\sum_{\alpha \in \mathbf{Z}^{s}} \psi(\alpha) \neq 0$. Observing that $S_{h}(\psi)=E^{h x} S_{h}(\varphi)$, we conclude that dist $\left(1, S_{h}(\psi)\right)$ $=\operatorname{dist}\left(1, S_{h}(\varphi)\right)$ for all $h$. As for $\psi$, this function is regular, hence Corollary (2.13) implies that dist $\left(1, S_{h}(\psi)\right) \neq O(h)$, unless $\psi *^{\prime} 1=$ const. $\neq 0$. Yet, the latter implies $\varphi *^{\prime} 1=$ const. $\neq 0$, which is impossible, since by (4.1), $\left.\left(\varphi *^{\prime} 1\right)\right|_{\chi^{s}}=0$.

To this end, we assume therefore that (4.2) holds and mention that already the authors of [SF] indicated that (4.2) does not contradict good approximation orders (as a matter of fact, [SF] identifies the "bad case" as being $\hat{\varphi}(0)=0$, a condition which is implied by (4.2)). Indeed, let $\varphi$ be a regular compactly supported function, and $p$ a linear polynomial, $p(1, \ldots, 1)=0$. Defining $\psi:=p(E) \varphi$, we see that $\psi$ is singular, yet $S(\varphi)=S(\psi)$, hence the approximation rates from the scales of $S(\psi)$ can be arbitrarily high, as the approximation rates associated with $S(\varphi)$ can.

In general, not every compactly supported singular spline is obtained by differencing a regular one (the results in the sequel will prove this indirectly). However, in the univariate case, every compactly supported function that satisfies (4.2) is obtained by differencing another function. This leads to the following

(4.4) Propositron. For a univariate $\varphi$, Theorem (3.27) holds even without the assumption $\sum_{\alpha \in \mathbf{Z}} \varphi(\alpha) \neq 0$. 
Proof. There is nothing to prove unless (4.1) holds. Moreover, if (4.2) does not hold, then Proposition (4.3) shows that both (a) and (b) of Theorem (3.27) are false for every positive $d$. We can therefore assume that (4.2) is valid. Now, choose $k$ such that supp $\varphi \subset[0, k)$. Invoking Proposition 3.2 of $\left[\mathbf{R}_{2}\right]$, we conclude that $\varphi=(E-1) \psi$ for some function $\psi$ supported in $[0, k-1)$. Since $S(\varphi)=S(\psi)$, we may replace $\varphi$ by $\psi$ for determining the approximation order. If $\psi$ satisfies $\psi *^{\prime} 1=0$, the process can be repeated. After at most $k-1$ steps, we obtain a function $\tau$ with $S(\tau)=S(\varphi)$, which necessarily satisfies $\tau *^{\prime} 1 \neq 0$, hence we are reduced to the former case.

We are aiming now at proving the following

(4.5) CLAIM. There exists a singular compactly supported function $\varphi$ for which the implication (b) $\Rightarrow$ (a) of Theorem (3.27) is invalid.

By the results so far, such $\varphi$ cannot be univariate, and must satisfy (4.2). We will not construct this $\varphi$ explicitly. Instead we draw here a link between our setting and the more involved case of several compactly supported functions, and make use of (negative) results known in the latter case.

The setting is as follows: $\Phi:=\left\{\varphi_{j}\right\}_{j=1}^{n}$ is a set of compactly supported functions. $S(\Phi)$ is the space spanned by their integer translates. An exponential space, here is a space $H$ which satisfies

$$
H \subset \sum_{\theta \in \Theta} e_{\theta} \Pi \text {, }
$$

for some finite $\Theta \subset \mathrm{C}^{s}$. The minimal possible $\Theta$ in (4.6) is the spectrum of $H$. An exponential is an element of an exponential space. For a compactly supported $\varphi$, we define $H(\varphi)$ as the space of all exponentials in $S(\varphi) . H(\varphi)$ is of importance in multivariate splines, since its local approximation properties may lead to lower bounds on the approximation order attained by appropriate refined versions of $S(\varphi)\left([\mathrm{DR}],\left[\mathrm{BR}_{3}\right]\right)$. Here, we always assume that $H(\varphi)$ is $D$-invariant, i.e., closed under differentiations; this implies that $H(\varphi)$ can be decomposed into

$$
H(\varphi)=\bigoplus_{0 \in \Theta} e_{\theta} P_{\varphi, \theta}
$$

where $P_{\varphi p, \theta}$ are finite-dimensional $D$-invariant polynomial spaces. Our result is as follows:

(4.8) THEOREM. Let $\Phi$ be as above and assume that $H\left(i_{j}\right)$ is D-invariant for $j=1, \ldots, n$. Then, for $s>1$, there exists a compactly supported $\psi \in S(\Phi)$ that satisfies

$$
H\left(\varphi_{j}\right) \subset H(\psi), \quad j=1, \ldots, n .
$$

First, we show how the above theorem is connected to Claim (4.5). In the above terminology, a polynomial space is a special case of an exponential space. For a polynomial space, $\left[\mathrm{B}_{1}\right]$ proves that $S(\varphi) \cap \Pi$ is always $D$-invariant, and so Theorem (4.8) shows in particular that there exists a compactly supported $\psi \in S(\Phi)$ which satisfies

$$
\text { (4.10) } \quad S\left(\varphi_{j}\right) \cap \Pi \subset S(\psi), j=1, \ldots, n \text {. }
$$

Now, the example given in $\left[\mathrm{BH}_{2}\right]$ provides an instance of a set $\Phi=\left\{\varphi_{1}, \varphi_{2}\right\}$ of two bivariate box splines such that $m_{3} \subset S(\Phi)$, yet the corresponding approximation order from $\left\{\sigma_{h} S(\Phi)\right\}_{h}$ is only 3 (and not 4 as might have been expected). By Theorem (4.8), there exists $\psi \in S(\Phi)$ satisfying

$$
\Pi_{3} \subset S(\psi)
$$

while the approximation order from $S_{h}(\psi)$ is at most 3 , since $S(\psi)$ is a subspace of $S(\Phi)$.

In the rest of this section we prove Theorem (4.8), and discuss other problems initiated by the theorem and related to the case of several $\varphi$ 's. The discussion of approximation orders for $\left\{S_{h}\right\}_{h}$ ends therefore at this point. Note that we were unable to comment on the validity of the implication $(a) \Rightarrow(b)$ in Theorem (3.27) in the case (4.2) is assumed.

We turn now to the proof of Theorem (4.8). This proof is based on the following result from $\left[\mathrm{BR}_{2}\right]$ :

(4.11) Result. Let $e_{\theta} P, P \subset \Pi$, be a D-invariant subspace of $S(\varphi)$, $\varphi$ being compactly supported. Then there exists a finite-dimensional $D$-invariant $Q \subset \Pi$ such that $e_{\theta} Q$ is mapped by $\varphi *^{\prime}$ onto $e_{\theta} P$.

The theorem now follows by a repeated application of the following lemma:

(4.12) LeMMA. Let $\varphi_{1}, \varphi_{2}$ be two compactly supported functions defined on $\mathbf{R}^{s}$ where $s>1$. Let $H_{1}, H_{2}$ be two D-invariant exponential spaces. If

$$
H_{j} \subset S\left(\varphi_{j}\right), \quad j=1,2,
$$

then there exists a compactly supported $\psi \in S\left(\left\{\varphi_{1}, \varphi_{2}\right\}\right)$ such that

$$
H_{j} \subset S(\psi), \quad j=1,2 .
$$

Proof. Set $\Theta_{j}$ for the spectrum of each $H_{j}$. Since each $H_{j}$ is $D$-invariant, it is a direct sum of spaces of the form $e_{\theta} P_{\theta}$, where $\theta \in \Theta_{j}$ and $P_{\theta}$ is a $D$-invariant polynomial space. Hence, by Result $(4,11)$, there exist finite-dimensional exponential spaces $F_{j}$ with spectrum $\Theta_{j}, j=1,2$, such that

$$
\dot{\varphi}_{j} *^{\prime} F_{j}=H_{j}
$$

Define $v^{1}:=(1,0, \ldots, 0), v^{2}:=(0,1,0, \ldots, 0)$. Let $T_{j}, j=1,2$, be two nontrivial finite difference operators such that, for $j=1,2$,

(a) $T_{j}$ is supported on $v^{j} \mathbf{Z}$;

(b) $T_{1} F_{2}=T_{2} F_{1}=0$. 
Each $T_{j}$ is a finite difference operator, and therefore for sufficiently high $k$, $T_{j}\left(e_{\theta} 0^{k \nu}\right) \neq 0$, for all $\theta \in \Theta_{1} \cup \Theta_{2}$, which implies that $T_{j} U_{j}$ is 1-1 on $e_{\theta} \Pi$ for each $\theta \in \Theta_{1} \cup \Theta_{2}$, where

$$
U_{j}: f \mapsto 0^{k v^{j}} f, \quad j=1,2 .
$$

Now, let $V_{j}$ be a finite difference operator which inverts $\left.\left(T_{j} U_{j}\right)\right|_{F_{j}}$ from the left, and define $G_{j}:=V_{j} U_{j} F_{j}$. Note that $T_{1}$ commutes with $V_{1}, V_{2}$ and $U_{2}$, hence

$$
T_{1} G_{2}=V_{2} U_{2} T_{1} F_{2}=0, \quad T_{1} G_{1}=T_{1} V_{1} U_{1} F_{1}=V_{1} T_{1} U_{1} F_{1}=F_{1},
$$
and similarly $T_{2} G_{1}=0, T_{2} G_{2}=F_{2}$. Define

$$
\psi=\sum_{j=1}^{2} T_{j} \varphi_{j}
$$

Then, by the above,

$$
\psi *^{\prime} G_{1}=\varphi_{1} *^{\prime}\left(T_{1} G_{1}\right)+\varphi_{2} *^{\prime}\left(T_{2} G_{1}\right)=\varphi_{1} *^{\prime} F_{1}=H_{1},
$$

and similarly $\psi *^{\prime} G_{2}=H_{2}$.

(4.15) CoRollary. Assume $s>1$. Then every finite-dimensional $D$-invariant exponential space is $H(\varphi)$ for some compactly supported $\varphi$.

Proof. From [R ; Thm. 1.1] we know that every finite-dimensional $D$-invariant exponential subspace of $e_{\theta} \Pi$ (with $\theta \in \mathbf{C}^{s}$ ) is $H(\varphi)$ for some compactly supported $\varphi$. Theorem (4.8) then completes the proof, since every finite-dimensional $D$-invariant exponential space is the sum of $D$-invariant spaces of the form $e_{\theta} P \subset e_{\theta} \Pi$.

The fact that the univariate case was not covered here is essential and is not related to the technique used (cf. Proposition 4.6 in $\left[R_{2}\right]$, which shows that not every univariate $D$-invariant exponential space is $H(\varphi)$ for some $\varphi$ ). For completeness, we record the following result, which under some "regularity" assumption (where regularity is now in the sense of $\left[R_{2}\right]$ ) enables us to obtain more, and in particular to include the univariate case.

(4.16) THEOREM. Let $\Phi=\left\{\varphi_{1}, \ldots, \varphi_{n}\right\}$ be a collection of $n$ compactly supported functions. For $j=1, \ldots, n$, let $H_{j}$ be a $D$-invariant exponential subspace of $S\left(\varphi_{j}\right)$ with spectrum $\Theta_{j}$ such that, for $j \neq k$,

$$
\left(\Theta_{j}-\Theta_{k}\right) \cap 2 \pi i \mathbf{Z}^{s}=\varnothing .
$$

Then there exists a compactly supported $\psi \in S(\Phi)$ with the properties

(a) $H_{j} \subset S(\psi), \forall j$

(b) $\psi *^{\prime} e_{\theta} \neq 0$, in case $\theta \in \Theta_{j}$ and $\varphi_{J} *^{\prime} e_{\theta} \neq 0$.

Proof. As in the previous proof; we first apply Result (4.11) to find exponential spaces $\left\{F_{j}\right\}_{j}$ with corresponding spectra $\left\{\Theta_{j}\right\}_{j}$ such that $\varphi_{j} *^{\prime} F_{j}=H_{j}$, $j=1, \ldots, n$. The assumption (4.17) then implies ([BAR], $\left.\left[\mathrm{BR}_{3}\right]\right)$ the existence of difference operators $\left\{T_{j}\right\}_{j}$ supported on $\mathbf{Z}^{s}$ such that

$$
T_{j} F_{k}=0 \text { for } j \neq k, \quad T_{j} \text { is } 1-1 \text { on } \sum_{\theta \in \Theta_{j}} e_{\theta} \Pi \text {. }
$$

Defining $\psi=\sum_{j} T_{j} \varphi_{j}$, we obtain

$$
\psi *^{\prime} F_{k}=\sum_{j} \varphi_{j} *^{\prime} T_{j}\left(F_{k}\right)=T_{k}\left(\varphi_{k} *^{\prime} F_{k}\right)=T_{k} H_{k} .
$$

Since $H_{k}$ is $D$-invariant, hence translation-invariant, $T_{k} H_{k} \subset H_{k}$. Yet, $T_{k}$ is 1-1 on $\sum_{\theta_{k}} e_{\theta} \Pi$, hence is also $1-1$ on its subspace $H_{k}$. We conclude that $T_{k} H_{k}=H_{k}$, and (a) follows.

For (b), we first note that whenever $j \neq k, T_{j} H_{k}=\varphi_{k} *^{\prime}\left(T_{j} F_{k}\right)=0$, by (4.18), and hence if $e_{\theta} \in H_{j}$ for some $j$, then, as in (4.19), $\psi *^{\prime} e_{\theta}=T_{j}\left(\varphi_{j} *^{\prime} e_{\theta}\right)$ Since $T_{j}$ is injective on $H_{j}, T_{j} e_{\theta}=c e_{\theta}$ for some nonzero $c$, so that

$$
\psi *^{\prime} e_{\theta}=c\left(\varphi_{j} *^{\prime} e_{\theta}\right), \quad c \neq 0,
$$

and (b) follows.

Corollary 2.1 of $\left[\mathrm{R}_{1}\right]$ shows that the above theorem is sharp in following sense: whenever $e_{\theta}, e_{\vartheta} \in S(\psi)$ and $\theta-\vartheta \in 2 \pi i \mathbf{Z}^{s} \backslash 0, \psi *^{\prime} e_{\theta}=\psi *^{\prime} e_{\vartheta}=0$.

Acknowledgement. It is my pleasure to thank Kirk Haller, Tom Hogan and Karol Dziedziul for their helpful remark concerning Theorem (3.23).

\section{References}

[BAR] A. Ben-Artzi and A. Ron, Translates of exponential box splines and their related spaces, Trans. Amer Math Soc. 309 (1988), 683-710.

$\left.\mathrm{B}_{1}\right] \mathrm{C}$. $\mathrm{de} \mathrm{Boor}$ The polynomials in the linear span of integer translates of a compactly supported function, Constr. Approx. 3 (1987), 199-208.

$\left.\mathrm{B}_{2}\right] \quad-$, Quasinterpolants and approximation power of multivariate splines, in: Computation of Curves and Surfaces, M. Gasca and C. A. Micchelli (eds.), Kluwer, Dordrecht 1990, 313-345.

BH $\left.{ }_{1}\right]$ C. de Boor and K. Höllig, B-splines from parallelepipeds, J. Analyse Math. 42 (1982/3), $99-115$.

$\left[\mathrm{BH}_{2}\right]-\ldots$, Approximation order from bivariate $\mathrm{C}^{1}$-cubics: a counterexample, Proc. Amer. Math. Soc. 87 (1983), $649-655$

[BJ] C. de Boor and R.-Q. Jia, Controlled approximation and a characterization of the locat approximation order, ibid. 95 (1985), 547-553.

$\left[\mathrm{BR}_{1}\right]$ C. de Boor and A. Ron, On multivariate polynomial interpolation, Constr. Approx. $6(1990), 287302$.

[BR $\left.\mathrm{BR}_{2}\right] \quad$ - Polynomial ideals and multivariate splines, in: Multivariate Approxination Theory V, W. Schemp \& K. Zeller (eds.), Birkhäuser, Basel 1990, 31 - 40.

CAR - . The exponentials in the span of the integer translates of a compactly supported function, CS TR 887, University of Wisconsin-Madison, November 1989, J. London Math. Soc., to appear.

[CD] C. K. Chui and H. Diam ond, A natural formulation of quasi-interpolation by multivariate splines, Proc. Amer. Math. Soc. 99 (1987), 643 646.

[CJW] C. K. Chui, K. Jetter and J. Ward, Cardinal interpolation by multivariate splines, Math Comp. 48 (1987), 711-724. 
$\left[\mathrm{DM}_{1}\right] \mathrm{W}$. Dahmen and C. A. Micchelli, Translates of mulivariate splines, Linear Algebra Appl. 52/3 (1983), 217-234.

$\left[\mathrm{DM}_{2}\right]-,-$, On the approximation order from certain multivariate spline spaces, J. Austral. Math. Soc. Ser. B 26 (1984), 233-246.

[DR] N. Dyn and A. Ron, Local approximation by certain spaces of multivariate exponentialpolynomials, approximation order of exponential box splines and related interpolation problems, Trans. Amer. Math. Soc. 319 (1990), 381-404.

[J] R.-Q. Ji a, A counterexample to a resull concerning controlled approximation, Proc. Amer. Math. Soc. 97 (1986), 647-654.

$\left[\mathrm{R}_{1}\right]$ A. Ron, Relations between the support of a compactly supported function and the exponential-polynomials spanned by its integer translates, Constr. Approx. 6 (1990), $139-155$.

$\left[\mathrm{R}_{2}\right] \quad-$, Factorization theorems for tninariate splines on regular grids, Israel J. Math. 70 (1990), 48-68.

[SF] G. Strang and G. Fix, A Fourier analysis of the finite element variational mothod, in: Constructive Aspects of Functional Analysis, G. Geymonat (ed.), C.I.M.E. II Ciclo 1971, Ed. Cremonese, Roma 1973, 793--840.

CENTER FOR THE MATHEMATICAL SCIENCES and

UNIVERSITY OF WISCONSIN-MADISON

\section{A note on Olech's Lemma}

by

ZVI ARTSTEIN* (Rehovot) and TADEUSZ RZEŹUCHOWSKI (Warszawa)

Abstract. A variant of Olech's Lemma in multifunctions integration is presented; it covers Abstract. A variant of Olies strong $L_{1}$-convergence.

We provide a version of the Olech Lemma concerning convergence to xtreme points in set-valued integration. Terminology and notations are recalled after the result is stated. We then compare our observation with the original Olech Lemma. After the proof is presented, we show how the new original Olech Lemme some compensated com pactness theory, of how weak convergence in $L_{1}$ may imply strong convergence.

The main result is as follows.

Proposrtron. Let $(\Omega, \mathscr{A}, v)$ be a measure space with $v$ an atomless, positive $\sigma$-additive measure. Let $F(\cdot)$ be a measurable $\mathbf{R}^{n}$ set-valued map with closed values. Let $e$ be an extreme point of $\int F(\omega) d v$. If $f_{k}(\cdot), k=1,2, \ldots$, is a uniformly integrable sequence of selections of $F(\cdot)$, and $\int f_{k}(\omega) d v$ converges to $e$, then the $f_{k}(\cdot)$ form a Cauchy sequence in $L_{1}\left(\Omega, \mathbf{R}^{n}\right)$. In particular, there exists a unique selection $e(\cdot)$ of $F(\cdot)$ such that $\int e(\omega) d v=e$, and the $f_{k}(\cdot)$ converge to $e(\cdot)$ in the $L_{1}\left(\Omega, \mathbf{R}^{n}\right)$ norm.

The terminology we use is standard, a good source is Castaing and Valadier [4]. For completeness we recall that $\int F(\omega) d v$ is defined as the set $\left\{\int f(\omega) d v: f(\cdot)\right.$ is integrable, and $f(\omega) \in F(\omega)$ for $v$-almost every $\left.\omega\right\}$. The set $\int F(\omega) d v$ is convex, since $v$ is atomless (see e.g. [4, Section IV.4]). A point $e$ is an extreme point of the convex set $C$ if $e=\frac{1}{2} a+\frac{1}{2} b$ with $a$ and $b$ in $C$ implies $e=a=b$. An extreme point of $C$ may not be an extreme point of $\mathrm{cl} C$, the closure of $C$, and this may be the case in the proposition, as $\int F(\omega) d v$ may not be a closed set.

The Olech Lemma is an extremely useful tool in the theory of existence and robustness of solutions to optimal control and variational problems; it was verified in Olech [5], see also Olech [6]. In the original version of the lemma, verified in Olech [5], see also Olech [6]. In the origin

1985 Mathematics; this work was * Incumbent of The Hettie H. Heinemann Professorial Chair in Humanities. 